\title{
Ciprofloxacin Resistant Shigella flexneri in India- A New Therapeutic Challenge
}

\author{
Neelam Taneja, Manisha Biswal, Pooja Rao, Garima Sangar, Prashant Sood, Shivapriya \\ Department of Medical Microbiology, \\ Postgraduate Institute of Medical Education and Research, Chandigarh, India
}

\begin{abstract}
Background \& Objective: Fluoroquinlones (FQ) have been highly effective drugs for treatment of shigellosis all over the world. Ciprofloxacin resistance in Shigella flexneri has emerged as a therapeutic challenge in our region. Here, we report clinical presentation of patients in whom $S$. flexneri was isolated from stool specimens as well as trends of ciprofloxacin susceptibility for the period 2000-2005.
\end{abstract}

Material and Methods: Stool samples were cultured and shigella spp. were identified using standard methods Antibiotic susceptibility was performed in accordance with Clinical Laboratory Standards (CLSI). Minimum inhibitory concentration (MIC) studies were performed by using the agar dilution technique of CLSI and E test. Patients' clinical details and response to therapy were noted. Plasmid profile of ciprofloxacin resistant strains was performed by the rapid alkaline lysis method. Conjugation experiments were done to determine whether quinolone resistance was transferable to $E$.coli J 53 Rif R.

Results : From 995 stool samples submitted from 1st Jan 2005 to 31st Dec 2005, 53 shigellae were isolated. S. flexneri (31 isolates, 60.7\%) was the predominant isolate, followed by $S$. dysenteriae (7), S. sonnei and S. boydii (6 each) and 3 (nonagglutinable). Isolates from 23 out of 28 patients (82\%) with S.flexneri shigellosis showed ciprofloxacin resistance (MIC>4). Seven patients infected with S.flexneri did not show any response to either ciprofloxacin/ ofloxacin, but 3 patients responded to ceftriaxone $\& 4$ patients respond to combination of amikacin $\&$ ciprofloxacin. Three patients showed a partial response, 2 relapsed after an initial response. Over a period of five years, a trend towards increasing MIC was noticed. Though the increase in MIC values appears gradual for MIC <4, a sharp peak is noticed for MIC> 4 in 2005. Though plasmids of 2, 4 and $1.8 \mathrm{~Kb}$ were transferred to $E$ coli, the $E$ coli conjugants were susceptible to ciprofloxacin, thereby confirming that ciprofloxacin resistance was not plasmid mediated

Interpretation \& Conclusion: There is a great immediate need for an effective oral agent that can be safely used for treatment of children with shigellosis along with continued surveillance required at regional and national level.

Keywords : Shigella flexneri, Ciprofloxacin resistance, India

\section{INTRODUCTION}

Fluoroquinlones (FQ) have been highly effective drugs for treatment of shigellosis all over the world. Ciprofloxacin resistance in Shigella flexneri is sporadic

\section{Corresponding Author :}

Dr. Neelam Taneja,

Department of Medical Microbiology,

Postgraduate Institute of Medical Edu. \& Research,

Chandigarh, India

Phone: 91-172-2742717; Fax 91-172-2744401

E-mail:drneelampgi@yahoo.com,
\& uncommon, although resistance to cotrimoxazole \& ampicillin is common and in some areas resistance to nalidixic acid has also emerged..$^{1-4}$ At our tertiary care referral centre in Chandigarh, northern India, which caters to a large population of 5 neighboring states, antibiotic resistance in Shigella is being constantly monitored. In 2003, there was an outbreak of ciprofloxacin resistant $S$. dysenteriae serotype $1^{5}$ which re-emerged as the predominant serotype after a gap of nearly a decade. There was a serogroup shift in 2004 when $S$. flexneri again became the predominant isolate. Ciprofloxacin resistance in $S$. flexneri has emerged as a 
therapeutic challenge in our region. Here, we report the clinical presentation of patients in whom S. flexneri was isolated from stool specimens as well as trends of ciprofloxacin susceptibility for the period 2000-2005.

\section{MATERIALAND METHODS}

Stool samples were obtained, at the discretion of the provider, from hospitalized patients with symptoms of diarrhea. They were cultured and shigellae were identified using standard methods. ${ }^{6} \quad$ Shigellae were confirmed by serotyping using antisera from DenkaSeiken (Japan). Antibiotic susceptibility was performed in accordance with Clinical Laboratory Standards (CLSI). ${ }^{7}$ Minimum inhibitory concentration (MIC) studies were performed by using agar dilution technique of $\mathrm{CLSI}^{7}$ and E test. Patients' clinical details and response to therapy were noted. We also performed MIC studies of the available $S$. flexneri strains $(n=116)$ isolated from 2000 to 2005 for ciprofloxacin. Plasmid profile of ciprofloxacin resistant strains was performed by the rapid alkaline lysis method. ${ }^{5}$ Conjugation experiments were done to determine whether quinolone resistance was transferable to $E$. coli $\mathrm{J} 53 \mathrm{Rif} \mathrm{R} .{ }^{8}$ Transconjugants were selected on Mueller- Hinton agar plates containing rifampicin $(100 \mathrm{mg} / \mathrm{l})$ alone and with ciprofloxacin $(8 \mathrm{mg} / \mathrm{l})$.

\section{RESULTS}

From the 995 stool samples submitted from 1st Jan 2005 to 31 Dec 2005, 53 shigellae were isolated. S. flexneri (31 isolates, 60.7\%) was the predominant isolate, followed by $S$. dysenteriae (7), S. sonnei and. S. boydii (6 each) and 3 (nonagglutinable). Thirty-one S.flexneri were isolated from 28 patients. On presentation, duration of illness ranged from 2 days to 9 months, (median 7 days and average 28 days). The clinical presentation was as follows -acute dysentery 19 , acute diarrhea 5 , chronic diarrhea 1 , chronic dysentery 1 and pseudomembraneous colitis 1 . Age of patients (male 20, female $8 ; 10$ adults and 18 children) ranged from 3 months to 60 years. All children were below 5 years of age and 11 were below 2 years. All patients were treated with ciprofloxacin / ofloxacin. Seven patients did not show any response to either ciprofloxacin/ ofloxacin, but 3 patients responded to ceftriaxone $\& 4$ patients respond to combination of amikacin \& ciprofloxacin. Three patients showed a partial response (responded to prolonged treatment with 10 days of fluroquinolone), 2 relapsed after an initial response. Sixteen patients were from Chandigarh and rest 12 were from the neighboring regions, 344 kilometers across in length, showing widespread distribution of resistance to ciprofloxacin.

The MIC values to ciprofloxacin were as follows: one strain each with MIC values $(\mu \mathrm{g} / \mathrm{ml})$ of $0.064,0.125$, 0.5 and 4 respectively; two strains each with MIC values of $0.25,6$ and 32 respectively; seven strains with MIC values of 16 and 14 strains with MIC of 8 . Isolates from 23 out of 28 patients (82\%) with S.flexneri shigellosis showed ciprofloxacin resistance (MIC>4). Thirteen resistance patterns were obtained out of which 15 isolates had resistance to amoxicillin, nalidixic acid, cotrimoxazole, ciprofloxacin \& norfloxacin pattern. By disc diffusion susceptibility testing the following resistance was observed: amoxicillin (69\%), nalidixic acid $(96.7 \%)$, cotrimoxazole 27/31, norfloxacin (80.6\%), ciprofloxacin (19.3\%) and chloramphenicol $(12.9 \%)$. Ciprofloxacin resistance was not adequately detected by disc diffusion. In fact norfloxacin disc diffusion testing was a more accurate predictor for high MIC values against ciprofloxacin. Over a period of five years, a trend towards increasing MIC was noticed. Though the increase in MIC values appears gradual for MIC <4, a sharp peak was noticed for MIC>4 in 2005.The other notable downward trend is seen in MIC values $<0.0325$ (Fig I).

\section{DISCUSSION}

Fluoroquinolones are extensively used and misused for many other illnesses in our region. Quinolone resistance is linked mainly to mutations located in the quinolone

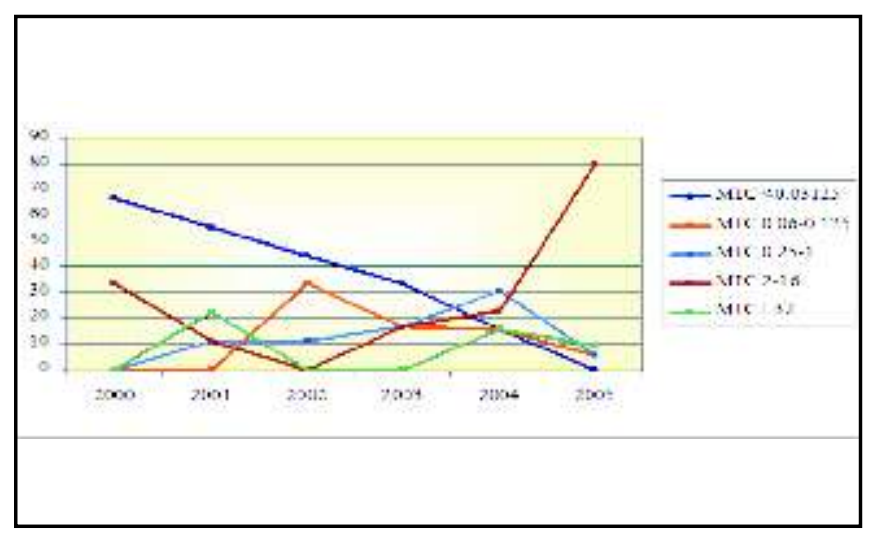

Fig. I: Decreasing susceptibility to ciprofloxacin in S. flexneri from 2000 to 2005 
resistance- determining regions (QRDRS) of DNA gyrase (gyrA and gyrB) and topoisomerase IV (par C and Par E). ${ }^{9}$ Recently, plasmid mediated fluoroquinolone resistance has been reported in Shigella. ${ }^{10}$ Though plasmids of 2, 4 and $1.8 \mathrm{~Kb}$ were transferred to E coli, the E coli conjugants were susceptible to ciprofloxacin, thereby confirming that ciprofloxacin resistance was not plasmid mediated. Treatment failures were earlier reported with ciprofloxacin resistant strains of $S$. dysenteriae 1 at our centre. ${ }^{5}$ Nalidixic acid resistance has been used as a marker for reduced susceptibility to ciprofloxacin in Salmonellae indicating possible first step gyr A mutations. ${ }^{11}$ However, as our strains were uniformly resistant to nalidixic acid, this disc could not be used to predict ciprofloxacin resistance. Disc diffusion susceptibility has limitations and therefore it is important to determine MIC. In our case, we found that resistance to norfloxacin disc could accurately detect ciprofloxacin resistance.

Unlike $S$. dysenteriae 1 which carries the maximum potential for epidemic spread, S. flexneri causes endemic shigellosis. Our past experience with $S$. flexneri has shown that $>60 \%$ cases presented with diarrhea and not dysentery. This time $68 \%$ of patients presented with frank dysentery. With drug resistant organisms there would be a delay in response and frank dysentery would develop later. But this alone cannot explain all the cases. Could these strains be more invasive? Could these strains be clonal? Antibiotic resistance has shown 15 strains belonging to one antibiogram. Plasmid profile of ciprofloxacin resistant strains showed eleven different patterns with common plasmids being of 2, 1.8, 1.2 and 4 $\mathrm{kb}$ sizes. Does this resistance have anything to do with ciprofloxacin resistance in $S$. dysenteriae 1 which occurred in 2003. Answering all these questions will require studies at molecular level. Also there is an immediate need for an effective oral agent that can be safely used for treatment of children with shigellosis along with continued surveillance required at regional and national level.

\section{REFERENCES}

1. Sivapalasingam S, Nelson JM, Joyce K, Hoekstra M, Angulo FJ,
Mintz ED. High prevalence of antimicrobial resistance among Shigella isolates in the United States tested by the National Antimicrobial Resistance Monitoring system from1999 to 2002. Antimicrob Agents Chemother 2006;50:49-54.

2. Yoshimura K, Kawashima I, Obana M, Matsuoka Y, Irimajiri S, Oonaka $\mathrm{K}$, et al. A case of bacillary dysentery caused by new quinolone-resistant Shigella flexneri 2a. Kansenshogaku Zasshi 1998;72(9):935-8.

3. Agniti MD, Soharno R, Lesmana M, Punjabi NH, Simanjuntak $\mathrm{C}$, Wangasaputra F, et al. The burden of diarrhoea, shigellosis and cholera in north Jakarta, Indonesia: findings from 24 months surveillance.BMC Infect Dis 2005;5:89.

4. Wang X Y, Du L, Von Seidlein L, Xu ZY, ZhangYL, Hao ZY, et al. Occurrence of shigellosis in the young and elderly in rural China: results of a 12 month population -based surveillance study. Am J Trop Med Hyg 2005;73:416-22.

5. Taneja N, Lyngdoh V, Vermani A, Mohan B, Rao P, Singh M et al. Re-emergence of multi-drug resistant Shigella dysenteriae with added resistance to ciprofloxacin in north India and their plasmid profiles. Indian J Med Res 2005;122:348-54.

6. World Health Organization. Manual for laboratory investigation of acute enteric infections, programme for control of diarrhoeal diseases. WHO CDD/ 83.3 Geneva, 1983.

7. Clinical and Laboratory Standards Institute. Performance standards for antimicrobial disk susceptibility tests. Approved standard, 9th ed. Document M2-A9.2006;CLSI, Wayne, Pa.

8. Martinez-Rodriguez J M, Pascual A, Garcia I, MartinezMartinez L. Detection of the plasmid-mediated quinolone resistance determinant qnr among clinical isolates of Klebsiella pneumoniae producing AmpC-type $B$-lactamase. J Antimicrob Chemother 2003;52:703-6.

9. Dutta S, KawamuraY, Ezaki T, Nair GB, Iida KI, Yoshida SI. Alteration in the gyr A subunit of DNA gyrase and the par C subunit of topoisomerase IV in quinolone -resistant S.dysenteriae serotype 1 clinical isolates from Kolkata, India. Antimicrob Agents and Chemother 2005;49:1660-1.

10. Hata M, Suzuki M, Matsumoto M, Takahashi M, Sato K, Ibe S and Sakae K. Cloning of a novel gene for quinolone resistance from a transferable plasmid in Shigella flexneri $2 \mathrm{~b}$. Antimicrob Agents and Chemother 2005;49:801-3.

11. Streit JM, Jones RN, Toleman MA, Stratchounski LS, Fritsche TR. Prevalence and antimicrobial susceptibility patterns among gastroenteritis-causing pathogens recovered in Europe and Latin America and Salmonella isolates recovered from bloodstream infections in North America and Latin America: report from the SENTRY Antimicrobial Surveillance program (2003). Int J Antimicrob Agents 2006;27:367-75. 